\title{
ESTUDO FITOQUÍMICO E AVALIAÇÃO DA ATIVIDADE ANTIBACTERIANA DE LAFOENSIA PACARI (LYTHRACEAE)
}

\author{
PHYTOCHEMICAL STUDY AND EVALUATION OF ANTIBACTERIAL \\ ACTIVITY OF LAFOENSIA PACARI (LYTHRACEAE)
}

\author{
Wellyson da Cunha Araújo Firmo ${ }^{1 ; 2}$, Milena Valadar Miranda ${ }^{1}$, Gizelli Santos \\ Lourenço Coutinho ${ }^{1}$, Luiz Mário da Silva Silveira ${ }^{3}$, Roberto Sigfrido Gallegos Olea ${ }^{4}$ \\ ${ }^{1}$ Mestre em Saúde e Ambiente pela Universidade Federal do Maranhão-UFMA, 65025-560, \\ São Luís-MA, Brasil.*E-mail: well firmo@hotmail.com \\ ${ }^{2}$ Docente da Faculdade de Educação de Bacabal-FEBAC, 65700-000, Bacabal-MA, Brasil. \\ ${ }^{3}$ Docente do Departamento de Farmácia da Universidade Federal do Maranhão-UFMA, \\ 65080-805, São Luís-MA, Brasil. \\ ${ }^{4}$ Docente do Departamento de Química da Universidade Federal do Maranhão-UFMA, \\ 65080-040, São Luís-MA, Brasil.
}

Data de recebimento: 07/05/2014

Data da aprovação: 12/06/2014

\section{RESUMO}

O uso de plantas medicinais é bastante antigo, este recurso natural é empregado para o tratamento das mais diversas doenças. A Lafoensia pacari é uma planta arbórea, conhecida popularmente por "mangaba brava", "pacari" ou "dedaleiro", usada na medicina tradicional brasileira para o tratamento do câncer, distúrbios gástricos, inflamação e cicatrização. A presente pesquisa tem como objetivo estudar o perfil fitoquímico e a atividade antibacteriana de L. pacari. Folhas da planta foram coletadas no município de Estreito-MA, das folhas secas foi obtido o extrato hidroalcoólico a 70\%, o qual foi submetido a testes fitoquímicos e ao de atividade antibacteriana utilizando a técnica de difusão em ágar (técnica do poço). Os resultados mostram a presença de compostos como: fenóis, taninos hidrolisáveis, flavanonóis, triterpenos, alcaloóides, saponinas, flavanonas, esteroides e terpenoides. A Staphylococcus aureus e Escherichia coli foram sensíveis em todas as concentrações testadas, sendo que o extrato foi mais sensível frente a $S$. aureus, possivelmente por ser uma bactéria gram positiva. Estes resultados mostram que a utilização deste extrato pode ser promissor em face de doenças ocasionadas por estes microorganismos, contudo são necessários estudos mais minuciosos.

Palavras-chave: Atividade antibacteriana. Lafoensia pacari. Plantas medicinais.

\begin{abstract}
Use of medicinal plants is quite old, this natural resource is used for the treatment of several diseases. Lafoensia pacari is a woody plant, popularly known as "mad mangaba", "pacari" or "dedaleiro", used in traditional Brazilian medicine for the treatment of cancer, gastric disorders, inflammation, and scarring. This research aim to study the phytochemical profile and the antibacterial activity of $L$. pacari. Leaves were collected in the municipality of Estreito-MA, the dried leaves alcoholic extract obtained was
\end{abstract}


$70 \%$, which was subjected to phytochemical tests and antibacterial activity using the agar diffusion technique (well technique). The results show the presence of compounds such as phenols, hydrolysable tannins, flavanonols, triterpenes, alkaloids, saponins, flavanones, steroids and terpenoids. The Staphylococcus aureus and Escherichia coli were sensitive at all concentrations tested, and the extract was more sensitive against S. aureus. These results show that the use of this extract may be promising for diseases caused by these microorganisms, however, it is necessary to more detailed studies.

\section{Keywords: Antibacterial activity. Lafoensia pacari. Medicinal plants. \\ 1 INTRODUÇÃO}

O conhecimento sobre as propriedades terapêuticas das plantas medicinais obtidas a partir da medicina popular vem sendo acumulado durante séculos, e esse conhecimento empírico simboliza muitas vezes o único recurso terapêutico de várias comunidades e grupos étnicos (ANGÉLICO, 2011).

As plantas medicinais constituem importantes recursos terapêuticos para o tratamento de doenças, principalmente das populações das nações em desenvolvimento. Servem tanto à conhecida "medicina caseira", que faz parte da cultura popular destes países, como de matéria-prima para elaboração de medicamentos fitoterápicos ou extração de compostos químicos com atividade terapêutica (FREITAS, 1999; CORDEIRO et al., 2006).

A diversidade de espécies de plantas presentes no Brasil continua representando um caminho para a descoberta de novos fármacos destinados ao tratamento de patologias que ainda hoje necessitam de inovações. A Organização Mundial de Saúde (OMS) vem apoiando a utilização de plantas medicinais por se tratar de uma prática tradicional em muitos povos e que traz benefícios para a saúde (SALVAGNINI et al., 2008; BONELLA et al., 2011).

Devido à resistência de patógenos aos antimicrobianos, é necessária a pesquisa de novos agentes para o combate de infecções. Drogas constituídas por extratos brutos ou compostos biologicamente ativos isolados de espécies vegetais usadas na medicina popular podem ser fontes promissoras para a pesquisa de novos fármacos antimicrobianos (AL-FATIMI et al., 2007). Estas substâncias também podem agir sinergicamente com outras, melhorando o potencial antibiótico (COUTINHO et al., 2004).

Entre essas espécies vegetais em estudo encontra-se Lafoensia pacari A. St.-Hil. (Lythraceae), que é uma planta arbórea, conhecida popularmente por "mangaba brava", "pacari” ou "dedaleiro", usada na medicina tradicional brasileira (GALDINO et al., 2009) para o tratamento do câncer, distúrbios gástricos, inflamação e cicatrização, utilizando as folhas e casca por métodos de infusão e maceração, sendo administrado principalmente pela via oral (SOLON et al., 2000). Encontrada na América Central e América do Sul, é observada no cerrado brasileiro (CABRAL; PASA, 2009), nos estados da Bahia, Goiás, Minas Gerais, Maranhão, Mato Grosso e Distrito Federal (CARVALHO, 1994).

Em estudos fitoquímicos, entre os principais metabólitos encontrados na L. pacari estão os da classe dos taninos, flavonoides, saponinas, esteroides, triterpenoides e alcaloides (VIOLANTE et al., 2009). Em outros trabalhos avaliando a atividade da espécie vegetal foi possível observar efeito antimicrobiano (LIMA et al., 2006), anti-inflamatório, analgésico (ROGERIO et al., 2006), antiúlcera (TAMASHIRO FILHO, 1999), antioxidante (SOLON et al., 2000) e antidepressivo GALDINO et al., 2009), o que demonstra o potencial farmacológico desta planta.

Desta forma, a presente pesquisa tem como objetivo estudar o perfil fitoquímico e a atividade antibacteriana de L. pacari.

\section{MATERIAIS E MÉTODOS}

\section{Coleta e preparo do extrato do material vegetal}

Folhas de L. pacari foram coletadas no Parque Nacional da Chapada das Mesas, no município de Estreito-MA, em julho de 2012. Uma exsicata foi preparada e depositada no Herbário Ático Seabra da Universidade Federal do Maranhão-UFMA, sob o número 1490. As folhas frescas (pelos menos 500g) foram levadas à estufa a $50^{\circ} \mathrm{C}$ durante 24 horas. Após 
secagem, as amostras foram trituradas em moinho, obtendo um pó moderadamente fino, o qual foi submetido à extração com álcool a $70 \%$ durante sete dias. O extrato foi concentrado sobre pressão reduzida em um evaporador rotativo e armazenado em frasco com rótulo e tampa de rosca.

\section{Triagem fitoquímica}

Os testes fitoquímicos foram realizados segundo a metodologia proposta por Matos (1997). O extrato e frações foi submetidos a testes para verificação de fenóis e taninos (reação com cloreto férrico), flavonoides (teste de variação de $\mathrm{pH}$, com hidróxido de sódio e ácido sulfúrico), esteroides e triterpenos (teste de Liebermann-Burchard), saponinas (teste de espuma e teste de precipitação), cumarinas (teste com luz UV), resinas (teste de turvação do látex) e alcaloides (identificação com Dragendorff, Hager e Mayer).

\section{Microorganismos}

Para a realização do teste de atividade antibacteriana foram utilizadas cepas padrão de Staphylococcus aureus (ATCC 25923) e Escherichia coli (ATCC 25922) pertencentes à bacterioteca do
Laboratório Central de Saúde Pública do Maranhão (LACEN-MA).

\section{Avaliação da atividade antibacteriana}

A avaliação da atividade antibacteriana do extrato foi realizada pelo método de difusão em ágar (técnica do poço).

\section{Método de difusão em ágar (técnica do poço)}

Foram confeccionados poços de $10 \mathrm{~mm}$ de diâmetro em placas contendo o meio ágar MuellerHinton (Merck). Os inóculos bacterianos foram semeados no meio de cultura e os poços preenchidos com $300 \mu \mathrm{L}$ do extrato nas concentrações 1000, 500, 250, 125 e $62,5 \mu \mathrm{g} / \mathrm{mL}$. Após o período de incubação de 24 horas, a $35^{\circ} \mathrm{C}$, mediu-se a zona de inibição ao redor do poço. $\mathrm{O}$ ensaio foi realizado em duplicata (SILVEIRA et al., 2007).

\section{RESULTADOS E DISCUSSÃO}

Na caracterização fitoquímica foi possível constatar grupos de compostos químicos provenientes do metabolismo secundário da planta. A Tabela 1 mostra os resultados obtidos na triagem a partir do extrato bruto hidroalcoólico $(\mathrm{EBH})$ do material vegetal.

Tabela 1 - Perfil fitoquímico do extrato bruto hidroalcoólico de Lafoensia pacari

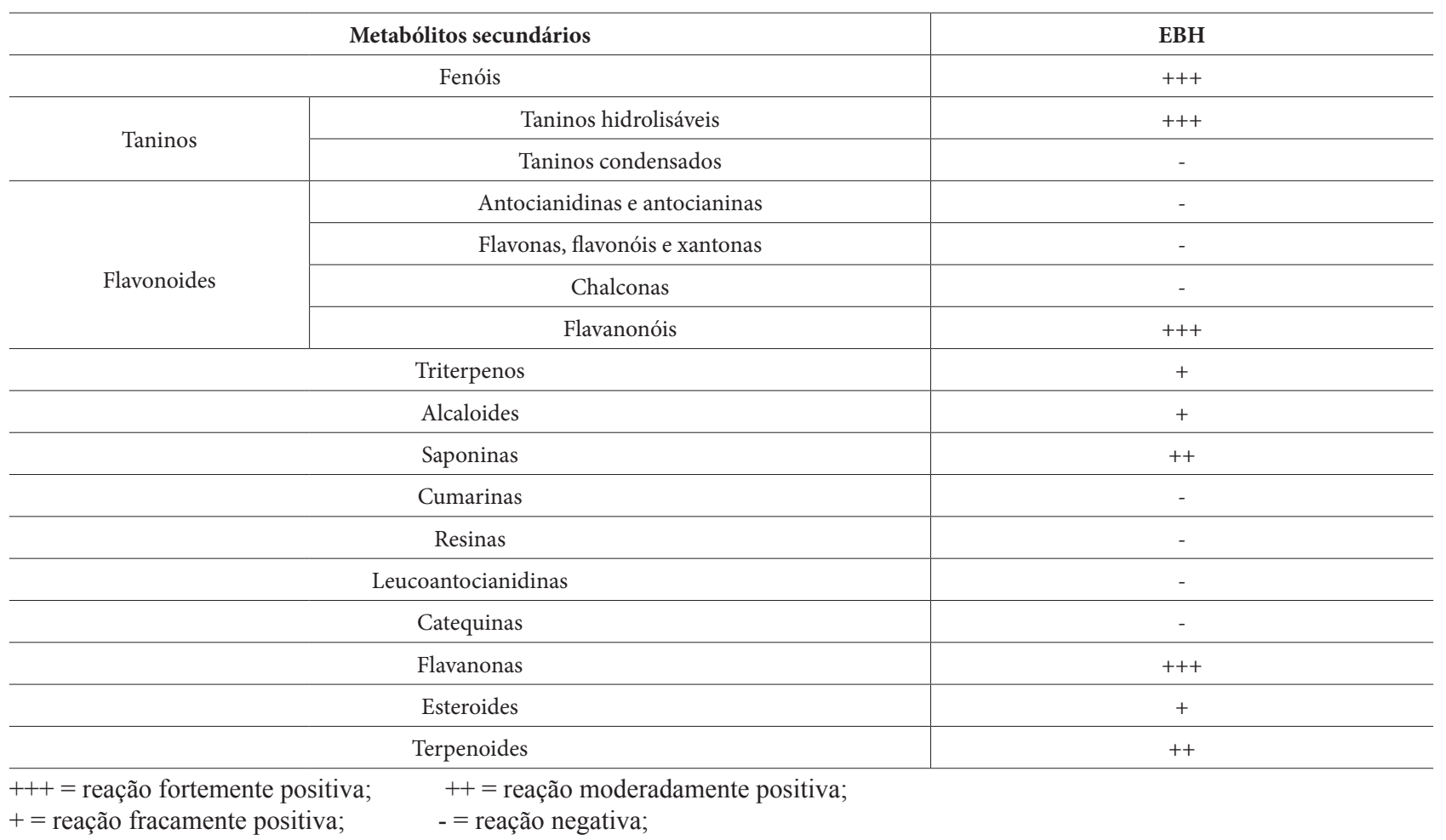


Entre os grupos químicos pesquisados neste trabalho, cujos resultados estão apresentados na Tabela 1 , é possível observar a positividade para fenóis, taninos (hidrolisáveis), flavanonóis, alcaloides, triterpenos, saponinas, flavanonas, esteroides e terpenoides.

No gênero Lafoensia, de acordo com Lorenzi (1992), encontram-se os principais compostos químicos da classe dos taninos, quinonas e principalmente alcaloides. Alguns trabalhos realizados com a espécie L. pacari, corroboram com a presente pesquisa ao evidenciar a presença de compostos ativos entre eles, taninos, flavonoides, saponinas, esteroides, triterpenoides e alcaloides (SANTOS; COELHO; PIRANI, 2009; VIOLANTE et al., 2009). Em pesquisa de Carvalho, Carvalho e Brás-Filho (1999), foram observados compostos ácidos, triterpenos, saponinas, flavonoides livres e glicosilados e acetofenonas. Lima et al. (2006) encontraram resultados positivos para taninos, fenóis, chalconas, auronas, flavonoides, leucoantocianidinas, antraquinonas e saponinas.
Os resultados desta pesquisa revelam a importância de estudos etnofarmacológicos, pois a L. pacari é usada popularmente como cicatrizante (TOLENTINO et al., 2011), tratamento de gastrite e úlceras (GUIMARÃES et al., 2010), e de inflamações (BUENO et al., 2005; CAMPOS; FRASSON, 2011), e estas ações são possíveis ou melhores quando há ocorrência de efeito antibacteriano, o que fez que essa atividade fosse investigada.

\section{CONCLUSÃO}

A análise fitoquímica revelou compostos do metabolismo secundário de L. pacari, importante para diversas ações e aplicações terapêuticas, muitas delas já aproveitadas pela população, sendo estes compostos potencialmente ativos em modelos biológicos e farmacológicos.

Os ensaios antibacterianos indicaram sensibilidade de ambas às bactérias frente ao extrato bruto hidroalcoólico, tendo maior efeito bactericida sobre S. aureus.

Tabela 2 - Atividade antibacteriana do Extrato Bruto Hidroalcoólico de Lafoensia pacari

\begin{tabular}{c|c|c|c|c}
\hline \multirow{2}{*}{ Microorganismos } & \multicolumn{4}{|c}{ Concentração $\boldsymbol{\mu g} / \mathbf{m L}$} \\
\cline { 2 - 5 } & $\mathbf{1 0 0 0}$ & $\mathbf{5 0 0}$ & $\mathbf{2 5 0}$ & $\mathbf{1 2 5}$ \\
\hline Escherichia coli & $18 \mathrm{~mm}$ & $16 \mathrm{~mm}$ & $14,5 \mathrm{~mm}$ & $13 \mathrm{~mm}$ \\
\hline Staphylococcus aureus & $22 \mathrm{~mm}$ & $20 \mathrm{~mm}$ & $19,5 \mathrm{~mm}$ & $17,5 \mathrm{~mm}$ \\
\hline
\end{tabular}

Os valores são médias de duas repetições

Em relação à atividade antibacteriana, observase na Tabela 2 que os resultados mostram significativa inibição do crescimento bacteriano sobre a $E$. coli e $S$. aureus, em todas as concentrações testadas, contudo nota-se que o EBH teve maior sensibilidade sobre a $S$. aureus. Isso deve-se possivelmente pela característica bacteriana desta espécie ser uma gram positivo, sendo mais fáceis a difusão e ação de substância antibacteriana.

Esta atividade pode estar relacionada à presença de compostos do metabolismo secundário desta planta, como fenóis, taninos hidrolisáveis, triterpenos, alcaloides, saponinas, flavanonas, esteroides e terpenoides, conforme observado na Tabela 1.

Estudos mostram a capacidade de plantas que apresentam composição química semelhante terem atividade antibacteriana, pois há relatos na literatura que descrevem atividades antimicrobianas destas classes de compostos (SINGH; SINGH, 2003; WENIGER et al., 2005; VALE; ORLANDA, 2011).
Devido à grande biodiversidade encontrada no Brasil, à etnofarmacologia e à importância de plantas no desenvolvimento de medicamentos, estudos como este são importantes para avaliar a eficácia de plantas medicinais, já que é um recurso natural bastante utilizado pela população.

\section{AGRADECIMENTOS}

Agradecemos à Fundação de Amparo à Pesquisa e Desenvolvimento Científico do Maranhão (FAPEMA), à Coordenação de Aperfeiçoamento de Pessoal de Nível Superior (CAPES), à Universidade Federal do Maranhão (UFMA), e ao Laboratório Central de Saúde Pública do Maranhão (LACEN-MA). 


\section{REFERÊNCIAS}

AL-FATIMI, M. et al. Antioxidant, antimicrobial and cytotoxic activities of selected medicinal plants from Yemen. Journal of Ethnopharmacology, v.111, p.657666, 2007.

ANGÉLICO, E. C. Avaliação das atividades antibacteriana e antioxidante de Croton heliotropiifolius KUNTE e Croton blanchetianus BAILL. 2011. 86f. Dissertação (Mestrado em Zootecnia). Universidade Federal de Campina Grande (UFCG). Patos-PB, 2011.

BONELLA, A. F. et al. Estudo fitoquímico e atividade antibacteriana de extratos de folhas de Acanthospermum australe (LOERFL.) In: KUNTZE. Enciclopédia Biosfera, Centro Científico Conhecer, v.7, n.13, p.1329-1335, 2011.

BUENO, N. R. et al. Medicinal plants used by the Kaiowá and Guarani indigenous populations in the Caarapó Reserve, Mato Grosso do Sul, Brazil. Acta Botanica Brasileira, v.19, n.1, p.39-44, 2005 .

CABRAL, P. R. F.; PASA, M. C. Mangava-brava: Lafoensia pacari A. St. -Hil. (Lythraceae) e a etnobotânica em Cuiabá, MT. Revista Biodiversidade, v.8, n.1, p.2-21, 2009.

CAMPOS, J. S.; FRASSON, A. P. Z. Avaliação da atividade antioxidante do extrato aquoso de Lafoensia pacari A. ST.-HIL. em emulsão não-iônica. Revista de Ciências Farmacêuticas Básica e Aplicada, v.32, n.3, p.363-368, 2011.

CARVAlHO, G. J. A.; CARVALHO, M. G.; BRÁSFILHO, R. A triterpenoid saponin isolated from Lafoensia glyptocarpa. Phytochemistry, v.52, p.1617-1619, 1999.

CARVALHO, P. E. R. Espécies florestais brasileiras: recomendações silviculturais, potencialidade e uso da madeira. Brasilia: EMBRAPA, CNPF. Colombo, 1994.

CORDEIRO, C. H. G. et al. Análise farmacognóstica e atividade antibacteriana de extratos vegetais empregados em formulação para higiene bucal. Revista Brasileira de Ciências Farmacêuticas, v.42, n.3, p.395-404, 2006.

COUTINHO, H. D. M. et al. Atividade antimicrobiana de produtos naturais. Revista Conceitos, v.10, n.10, p.77-85, 2004.

FREITAS, P. C. D. Atividade antioxidante de espécies medicinais da família Piperaceae: Pothomorphe umbellata (L) Miq e Piper regnellii (Miq) CDC. 1999. 115f. Tese (Doutorado em Fármaco e Medicamentos). Faculdade de Ciências Farmacêuticas, Universidade de São Paulo. São Paulo, 1999.

GALDINO, P. M. et al. Antidepressant-like effect of Lafoensia pacari A. St.-Hil. ethanolic extract and fractions in mice. Journal of Ethnopharmacology, v.124, p.581$585,2009$.
GUIMARÃES, H. A. et al. Effects of ethanolic extract of leaves of Lafoensua pacari A. St.-Hil., Lythraceae (pacari), in pain and inflammation models. Revista Brasileira de Farmacognosia, v.20, n.3, p.328-333, 2010.

LORENZI, H. Árvores brasileiras: manual de identificação e cultivo de plantas nativas do Brasil. Nova Odessa: Plantarum, 1992. p. 352

LIMA, M. R. F. et al. Anti-bacterial activity of some Brazilian medicinal plants. Journal of Ethnopharmacology, v.105, p.137-147, 2006

MATOS, F. J. A. Introdução à fitoquímica experimental. 2.ed. Fortaleza: EUFC, 1997. p.141

ROGERIO, A. P. et al. Anti-inflammatory, analgesic and antioedematous effects of Lafoensia pacari extract and ellagic acid. J Pharm Pharmacol, v.58, p.1265-1273, 2006.

SALVAGNINI, L. E. et al. Avaliação da atividade antibacteriana de folhas de Myrtus communis L. (Myrtaceae). Revista Brasileira de Farmacognosia, v.18, n.2, p.241244, 2008.

SANTOS, L. W.; COELHO, M. F. B.; PIRANI, F. R. Fenologia de Lafoensia pacari A. St.-Hil. (Lythraceae) em Barra do Garças, Mato Grosso, Brasil. Revista Brasileira de Plantas Medicinais, v.11, n.1, p.12-17, 2009.

SOLON, S. et al. Schmeda-Hirschmann G. Free radical scavenging activity of Lafoensia pacari. Journal of Ethnopharmacology, v.72, p.173-178, 2000.

SILVEIRA, L. M. S. et al. Atividade antibacteriana de extrato de gervão frente cepas de Staphylococcus aureus oxacilina-sensíveis e oxacilina-resistentes isoladas de amostras biológicas. RBAC, v.39, n.4, p.299-301, 2007.

SINGH, B.; SINGH, S. Anticrobial activity of terpenoids from Trichodesma amplexicaule Roth. Phytother Res., v.17, n.7, p.814-6, 2003.

TAMASHIRO FILHO, P. Avaliação da atividade antiúlcera do extrato bruto metanólico de Lafoensia pacari St. Hil. (Mangava brava). 1999. Dissertação (Mestrado em Saúde e Ambiente). Cuiabá: Universidade Federal de Mato Grosso, 1999.

TOLENTINO, T. A. et al. Efeito alelopático e atividade antioxidante de Lafoensia pacari. In: REUNIÃO ANUAL DA SOCIEDADE BRASILEIRA DE QUÍMICA, 34., 2011, Florianópolis. Resumos. Florianópolis: Sociedade Brasileira de Química, 2011.

VALE, V. V.; ORLANDA, J. F. F. Atividade antimicrobiana do extrato bruto etanólico das partes aéreas de Euphorbia tirucalli Linneau (Euphorbiaceae). Scientia plena, v.7, n.4, p.1-6, 2011. 
VIOLANTE, I. M. P. et al. Avaliação in vitro da atividade fotoprotetora de extratos vegetais do cerrado de Mato Grosso. Revista Brasileira de Farmacognosia, v.19, n.2A, p.452-57, 2009.

WENIGER, B. et al. Bioactive triterpenoids from Vochysia pacifica interact with cyclic nucleotide phosphodiesterase isozyme PDE4. Phytother Res., v.19, p.75-7, 2005. 